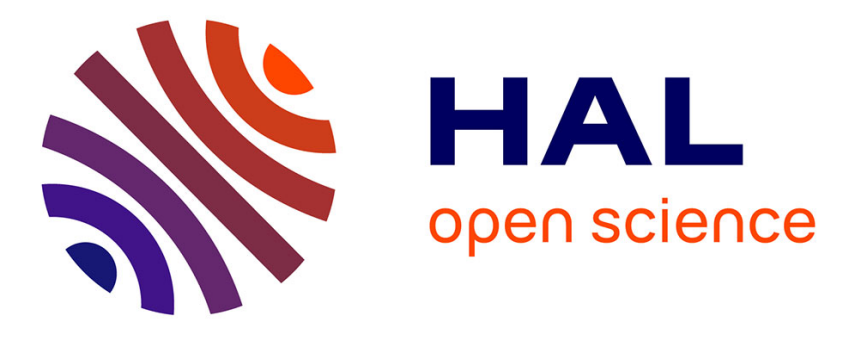

\title{
EBSD-assisted Laue microdiffraction for microstrain analysis
}

Taylan Örs, Jean-Sebastien Micha, Nathalie Gey, Vincent Michel, Olivier Castelnau, René Guinebretière

\section{- To cite this version:}

Taylan Örs, Jean-Sebastien Micha, Nathalie Gey, Vincent Michel, Olivier Castelnau, et al.. EBSDassisted Laue microdiffraction for microstrain analysis. Journal of Applied Crystallography, 2018, 51 (1), pp.55-67. 10.1107/S1600576717017150 . hal-02019196

\section{HAL Id: hal-02019196 https://hal.science/hal-02019196}

Submitted on 19 Feb 2019

HAL is a multi-disciplinary open access archive for the deposit and dissemination of scientific research documents, whether they are published or not. The documents may come from teaching and research institutions in France or abroad, or from public or private research centers.
L'archive ouverte pluridisciplinaire HAL, est destinée au dépôt et à la diffusion de documents scientifiques de niveau recherche, publiés ou non, émanant des établissements d'enseignement et de recherche français ou étrangers, des laboratoires publics ou privés. 
JOURNAL OF

APPLIED

CRYSTALLOGRAPHY

ISSN 1600-5767

Received 23 July 2017

Accepted 28 November 2017

Edited by A. Borbély, Ecole National Supérieure des Mines, Saint-Etienne, France

Keywords: Laue microdiffraction; electron backscatter diffraction; EBSD; microstrain analysis; zirconia; X-ray diffraction.

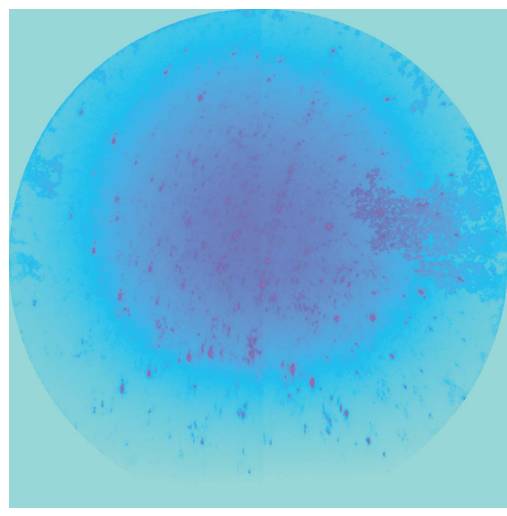

(C) 2018 International Union of Crystallography

\section{EBSD-assisted Laue microdiffraction for microstrain analysis}

\author{
Taylan Örs, ${ }^{\mathrm{a}, \mathrm{b} *}$ Jean-Sébastien Micha, ${ }^{\mathrm{c}}$ Nathalie Gey, ${ }^{\mathrm{b}}$ Vincent Michel, ${ }^{\mathrm{a}}$ Olivier \\ Castelnau $^{\mathrm{a}}$ and René Guinebretiere ${ }^{\mathrm{d} *}$
}

aPIMM, UMR CNRS 8006, ENSAM, CNAM, 151 boulevard de l'Hôpital, Paris, 75013, France, 'bEM3, UMR CNRS 7239 , Université de Lorraine, 7 rue Felix Savart, Cedex 1, Metz, 57045, France, ' CRG-IF BM32, Beamline at the European Synchrotron (ESRF) and Grenoble Alps University UMR SYMMES CEA/Grenoble/ Institut of Nanosciences and Cryogenics, 17 rue des Martyrs, Cedex 9, Grenoble, 38054, France, and ${ }^{\mathbf{d}}$ SPCTS, UMR CNRS 7315, Université de Limoges, 12 rue Atlantis, Limoges, 87068, France. *Correspondence e-mail: taylan.ors@ensam.eu, rene.guinebretiere@unilim.fr

The X-ray Laue microdiffraction ( $\mu$ Laue) technique has been establishing itself as a reliable means for microstrain analysis for the past few decades. One problem with this technique is that when the crystal size is significantly smaller than the probed volume and when the diffracting crystals are closely oriented, a large number of individual $\mu$ Laue patterns are superimposed in a complex way on the recorded diffraction images. In that case, because of the difficulty of isolating unambiguously a single-grain $\mu$ Laue pattern, a reliable analysis of strains is tedious manually and hardly achievable with current automated methods. This issue is even more severe for low-symmetry crystals or when highenergy X-rays are used, since each single-crystal $\mu$ Laue pattern already contains a large number of spots. This paper proposes overcoming this challenge through the development of a combined approach coupling $\mu$ Laue and electron backscatter diffraction (EBSD). The capabilities of this 'EBSD-assisted $\mu$ Laue' automated method are illustrated on a monoclinic zirconia-based specimen and $\mu$ Laue diffraction patterns are analysed with the crystal orientation input from EBSD. The obtained results are statistically reliable, reproducible and provide a physical insight into the micromechanical characteristics of the material.

\section{Introduction}

Information on the state of deformation within specimens at the sub-micrometre scale is required for many micromechanical studies (activation of slip systems and twinning, stress localization at grain boundaries and in precipitates, evaluation of the stored energy, damage initiation, crack propagation etc.). Examples of applications include stress concentration analyses within the grains (Spolenak et al., 2003; Wan et al., 2016), deformation mechanisms (Kirchlechner, Keckes, Micha et al., 2011; Pantleon et al., 2014; Jiang et al., 2015), observation of individual dislocations through a crystal (Clark et al., 2015; Hofmann et al., 2013) and even characterization of the elasto-plastic behaviour of the material at a (sub)micrometre scale (Plancher et al., 2017).

Generally speaking, the measurement of elastic strain in polycrystalline materials by diffraction can be done either by using a large beam probing simultaneously a large number of crystals or by using a microbeam with a size close to or smaller than that of each crystal and scanning the region of interest (ROI) within the sample. In the first case, the obtained values are averaged over many crystals, while in the second approach the extraction of information representative of the global sample requires a number of measurements realized step by 
step but microstrains in each crystal are directly shown. It is noticeable that in both cases the atomic displacement field and strain distribution are extracted at a spatial resolution lower than the crystal size. But in the first case the description of the strain profile is realized in the reciprocal space (see for example Boulle et al., 2003, 2005), whereas in the second case it is usually realized in the direct space through sample mapping, although distortions at a scale lower than the microbeam can be shown in the reciprocal space by the analysis of the Laue spot shape. Microdiffraction approaches rely on the development of microbeams and are common in electron-diffraction-based techniques. The development of third-generation synchrotron sources and advanced X-ray optics, on the other hand, has provided access to very small poly- or monochromatic X-ray beams.

The measurement of displacement fields can be obtained with very high spatial resolution close to the interatomic distances through high-resolution transmission electron microscopy (see e.g. Hÿtch et al., 2003). However, in such an approach the sample preparation clearly induces stress relaxation. Electron backscatter diffraction (EBSD) has become a very powerful method to measure orientation maps. Using scanning electron microscopy (SEM) with a field emission gun, the spatial resolution of EBSD can be as high as a few tens of nm (Humphreys, 2001). Using the high-angularresolution version of EBSD (HR-EBSD), the measurement of relative elastic strain becomes possible with a relative accuracy of $\sim 10^{-4}$ in favourable cases (Wilkinson et al., 2006; Villert et al., 2009; Plancher et al., 2016). EBSD patterns measured at two points corresponding to a close crystal orientation can be compared with each other by the digital image correlation (DIC) or cross-correlation (Wilkinson et al., 2006) techniques. To achieve reliable strain values, the orientation discrepancy between these two points should be smaller than $\sim 1^{\circ}$, although attempts to improve this limitation have been made (Maurice et al., 2012; Plancher et al., 2016). Apart from that, the strain values obtained are relative to a given reference point in the crystal. To obtain the absolute strain, the reference point must be taken from an area in the specimen known to be free of strain. In most materials, however, finding such a region beyond doubt is impossible. Measurement of absolute strain with HR-EBSD has been proposed through simulation of the EBSD pattern (Kacher $e t$ al., 2009), but limitations to this technique have also been pointed out (Maurice et al., 2010). The errors in strain measurements can be larger than $5 \times 10^{-3}$. In addition, the HR-EBSD technique is very demanding in terms of sample surface preparation.

Local elastic strain can also be measured by Kossel diffraction which was adapted to SEM in the 1970s (Dingley, 1975; Bouscaud et al., 2014). As the electron beam hits the material, the atoms generate X-rays through the fluorescence mechanism. These X-rays are diffracted by the crystal and create diffraction cones. One can detect these cones on a twodimensional detector and compare the measured $d$ spacings with an unstrained reference pattern or with a simulation with ideal lattice parameters of the same material. The spatial resolution $(\sim 1 \mu \mathrm{m})$ is significantly lower than that of HREBSD, the interaction depth being highly dependent on the material being analysed. The method is limited to materials with atomic numbers larger than $\sim 20$ in order to satisfy Bragg's condition for most lattice spacings. On top of that, the signal-to-noise ratio of the Kossel pattern is generally very low $(\sim 2 \%)$ and the acquisition time is significant (several minutes).

As stated above, $\mathrm{X}$-ray microbeams are accessible nowadays at all of the third-generation synchrotron radiation sources around the world (see e.g. Riekel, 2000). Two approaches have been developed, using either a mono- or a polychromatic beam. In the first case, coherent diffraction allows determination of the strain field within an isolated single crystal of sub-micrometre size (Robinson \& Harder, 2009). To overcome the sample size constraint in the standard coherent diffraction methods, X-ray ptychography has been developed (Godard et al., 2011); although this is a highly promising technique, because of the difficulty in phase retrieval of the diffracted beam it has only been applied to quasi-defect-free single-crystalline regions. Monochromatic $\mathrm{X}$-ray beams are also used in so-called 'three-dimensional X-ray diffraction microscopy' (Oddershede et al., 2010; Poulsen, 2012), where near- and far-field detectors are used to obtain three-dimensional information on the microstructure of the specimen. This technique is based on transmission diffraction geometry and therefore requires synchrotron radiation for most bulk samples. It is reported to be able to measure strains with a resolution in the range $8-20 \times 10^{-5}$ with a far-field detector (Oddershede et al., 2010). The main limitations of this technique are the low spatial resolution ( $10 \mu \mathrm{m}$ at best), calibration of the complex setup parameters (Borbély et al., 2014) and the overlapping of the reflections in the case of a crystal exhibiting high mosaicity.

The second X-ray microbeam approach, on which the present paper is focused, concerns the use of polychromatic radiation and is thus often called Laue microdiffraction ( $\mu$ Laue) (Chung \& Ice, 1999; Tamura et al., 2002; Hofmann \& Korsunsky, 2014). Nowadays, it is available at a few synchrotron sources around the world (Liu \& Ice, 2014; Ulrich et al., 2011; Chen et al., 2016), with software analysis tools such as LaueTools (Robach et al., 2014), LaueGo (Liu et al., 2004) and $X M A S$ (Tamura, 2014). The beam is focused down to submicrometre sizes with the help of highly focusing KirkpatrickBaez mirrors. The sub-micrometre beam size enables one to obtain diffraction patterns from a single-crystalline region for grain sizes within the micrometre range. Since the diffracted volume is of the order of a few $\mu \mathrm{m}^{3}$, using a polychromatic beam allows observation of multiple reflections at a given position of a single crystal without having to rotate the specimen as for monochromatic setups (Ungár et al., 2007). In that case, the technique is well established and has been shown to be very powerful (MacDowell et al., 2001; Kirchlechner, Keckes, Motz et al., 2011). Improvement of Laue image processing using DIC has been recently proposed by Petit $e t$ al. (2015) and Zhang et al. (2015), leading to excellent relative strain accuracy up to $10^{-5}$ (Zhang et al., 2017). Taking into 
account the cross section of the X-ray beam, the size of the beam imprint on the sample is of the order of $0.1-0.25 \mu \mathrm{m}^{2}$, and this is expected to decrease even further as a result of advances in X-ray beam focusing technologies. Depending on the nature of the sample, the penetration depth of the X-ray beam is between a few micrometres and a few tens of micrometres. Although the technique is routinely employed and very promising, there are still a number of difficulties to overcome with $\mu$ Laue.

First, for specimens with grains much smaller than the diffraction volume, a high density of Laue spots is observed on the detector screen, due to the superposition of many $\mu$ Laue patterns. This becomes even more apparent when the crystal structure exhibits a low symmetry, as each crystal already produces many spots, or similarly when high X-ray energies are used. To illustrate the difference between single-crystal and polycrystalline $\mu$ Laue diffraction, two patterns are shown in Fig. 1. The first one (Fig. 1a) is for a Ge single crystal, which produces only a few Laue spots; each spot exhibits a single intensity maximum (see Fig. 1c), and its shape can be well described by simple analytical functions such as a twodimensional Gaussian. The second pattern (Fig. 1b) is for a $\mathrm{ZrO}_{2}$ specimen with very small grains (microstructure details will be given in \$4) producing many spots; here most spots contain many intensity sub-maxima coming from several crystals in the diffraction volume. These sub-maxima are typically $0.5-1.5^{\circ}$ away from each other, corresponding to distances of 10-30 pixels on the detector, as seen in Fig. 1(d). Distributions of the angles between each of the observed diffraction vectors for both patterns are given in Figs. 1(e) and $1(f)$. The single-crystal $\mu$ Laue pattern contains far fewer peaks, and consequently the angles between diffraction vectors show a discrete distribution with each discrete angle being observed $\sim 10-15$ times (Fig. 1e). The standard indexing technique relies on comparing this distribution with the one calculated in advance for a strain-free crystal lattice. By testing a number of peak pair angles, it is relatively easy to determine the $(h k l)$ Miller index corresponding to each experimental peak (so-called indexing step), assuming the crystal structure is known. When the diffraction volume contains many crystals, the distribution of peak pair angles becomes a very dense and almost continuous function, with each angular value being observed a few hundred times even within a small tolerance angle (Fig. 1f). This has two consequences: (i) the CPU time necessary for indexing increases tremendously, as it is proportional to the square of the number of spots considered; (ii) more importantly, the probability of wrongly assigning together two peaks belonging to two different crystals increases severely. Consequently, the standard automatic indexing method is not appropriate anymore.
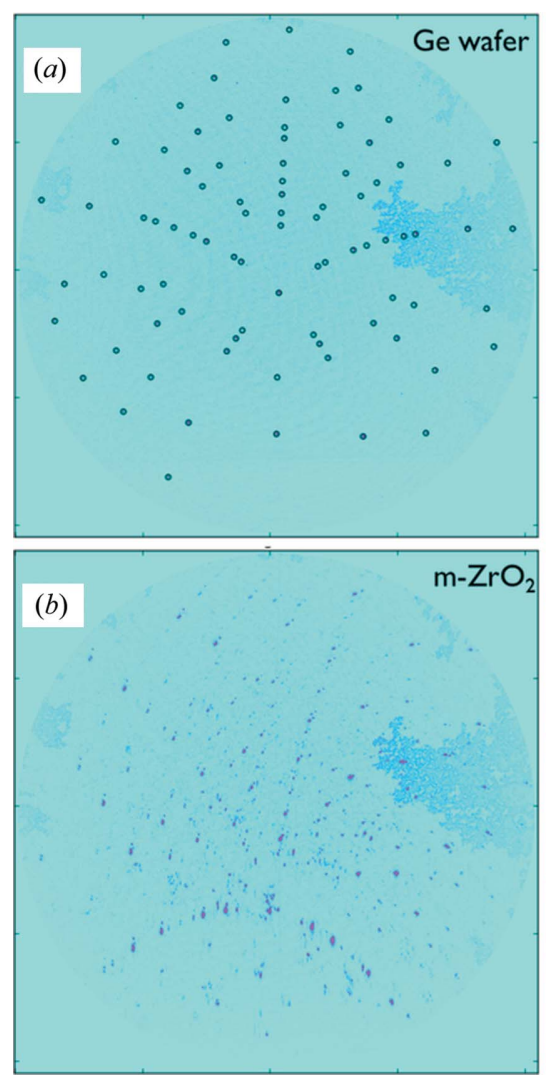
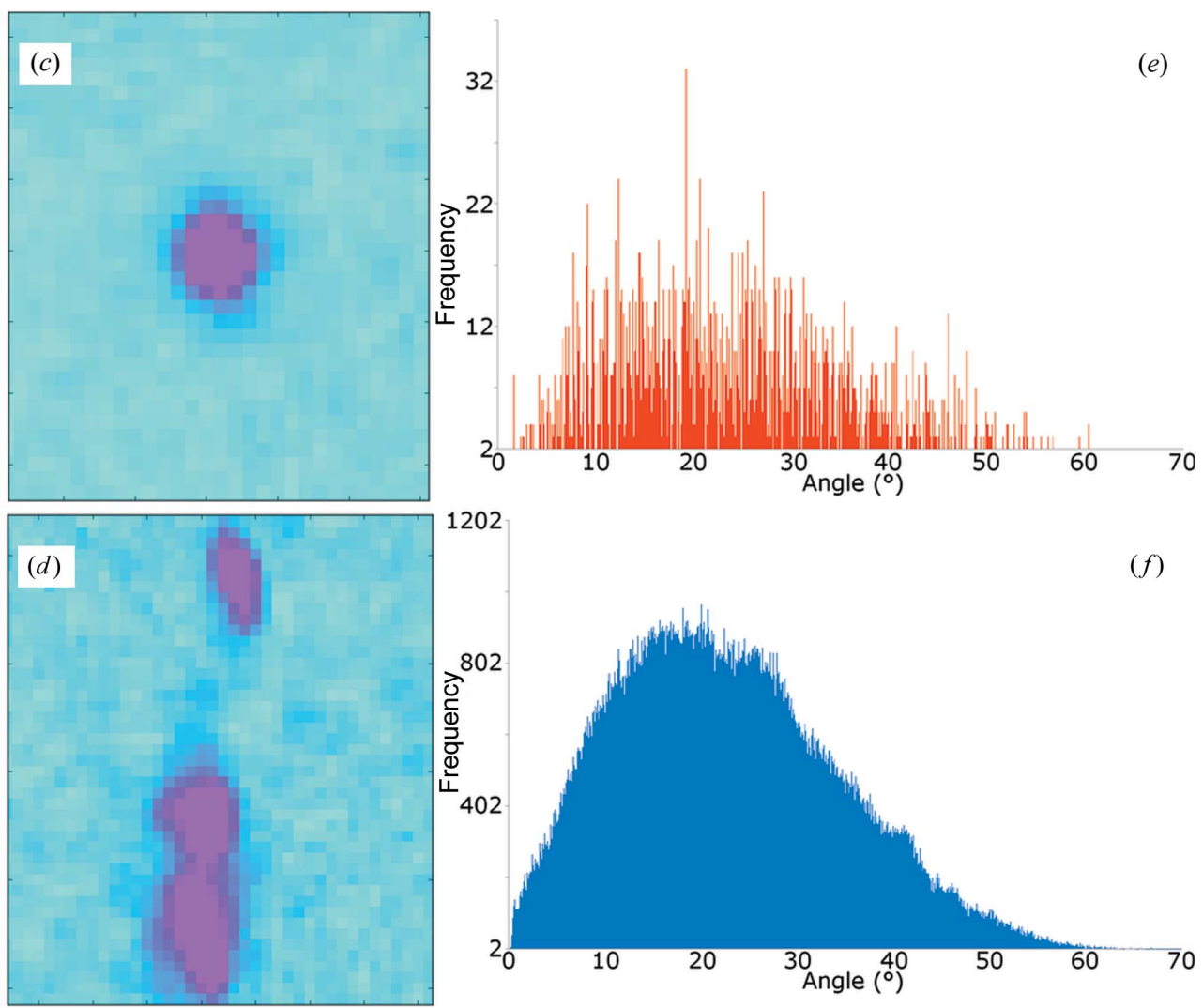

Figure 1

Observed $\mu$ Laue patterns of $(a)$ a single-crystal Ge wafer and $(b)$ a polycrystalline region on a monoclinic $\mathrm{ZrO}_{2}$ sample. Peak positions for the Ge wafer are circled for improved visibility. Close-ups of the patterns in $(a)$ and $(b)$ are given in $(c)$ and $(d)$, showing the difference in the observed peak shapes. $(e)$ and $(f)$ are the distributions of angle between the diffraction vectors obtained from patterns $(a)$ and $(b)$, respectively. The interval for the distribution bars is chosen as $0.1^{\circ}$ and the angular range is $0-70^{\circ}$. For clarity, angles observed at least twice are plotted. 
Second, owing to the penetration of the X-ray beam within the specimen, one cannot distinguish from the $\mu$ Laue pattern whether the diffracting crystal is located at the sample surface or somewhere deeper inside the specimen along the incoming beam, unless the time-consuming differential aperture X-ray microscopy technique is applied (Larson et al., 2002, 2004; Ohashi et al., 2009; Marijon, 2017). Sorting out grains lying at the specimen surface is of importance when one wants to make the link between local stresses measured by $\mu$ Laue and other microstructural or mechanical characterizations with laboratory techniques adapted to surface analyses, such as optical or electronic microscopies (Plancher et al., 2017).

In this work, we aim to push the limits of $\mu$ Laue one step forward in terms of robustness with respect to complex smallscale microstructures. We propose an 'EBSD-assisted $\mu$ Laue' method to index the $\mu$ Laue patterns and to measure elastic strain in materials exhibiting a complex sub-micrometre microstructure with a number of individual crystals within the gauge volume. In this approach, the indexing challenge concerning complex $\mu$ Laue images made of several superimposed diffraction patterns is solved by providing the $\mu$ Laue processing software with the orientation of the diffracting crystals of the gauge volume as a priori knowledge. This allows one to start the indexing procedure with an efficient guess for orientation and strain refinement. So far no such study has been carried out on a method that combines the high spatial resolution of EBSD and high angular (and therefore high strain) resolution of $\mu$ Laue.

To illustrate the proposed method, we make use of $\mu$ Laue data obtained on a fused-cast zirconia-based specimen. This material is a perfect case of a twinned microstructure with multiscale crystal sizes, down to nanometre scale, inherited from successive structural phase transformations (SPTs) that occur during cooling (Humbert et al., 2010). On top of that, the

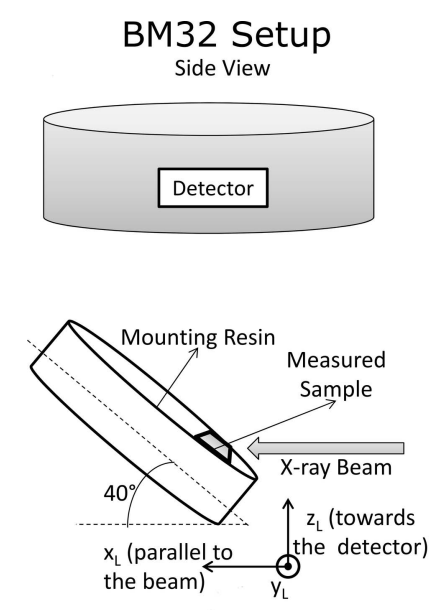

(a)

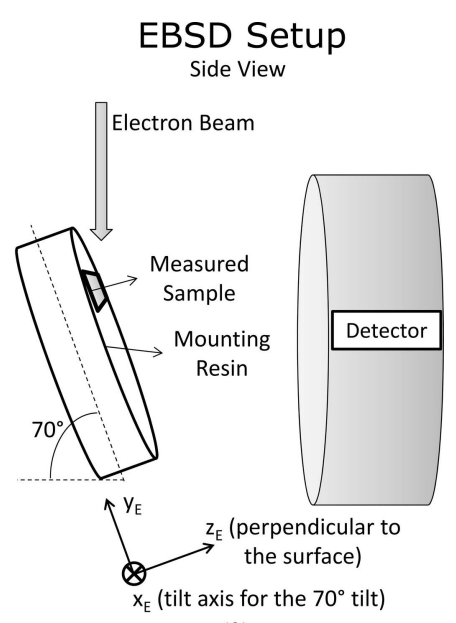

(b)
Figure 2

Experimental setup configurations of (a) $\mu$ Laue at BM32, ESRF (Grenoble, France), and (b) EBSD in SEM. The sample position and reference frames for crystal orientation definition are indicated on each setup. The reference frames labelled with indices $\mathrm{E}$ and $\mathrm{L}$ refer to EBSD and $\mu$ Laue setups, respectively. low-symmetry crystal structure (monoclinic) generates $\mu$ Laue patterns crowded by numerous spots.

After some general description of the conventional $\mu$ Laue approach ( $\$ 2)$, we describe in $\$ 3$ the method that we propose to label as EBSD-assisted Laue microdiffraction. Then, in $\S 4$, an illustrative application is presented. Finally, we discuss the performance of the method in terms of strain measurement for materials exhibiting sub-micrometre microstructures.

\section{Conventional Laue microdiffraction}

A standard $\mu$ Laue setup in reflection geometry consists of the following main parts: X-ray mirrors to focus the beam, a tilted sample stage and an area detector, as shown in Fig. 2(a). The stage is moved over an ROI and $\mu$ Laue patterns are acquired at each position. The conventional analysis of each $\mu$ Laue pattern goes through three successive steps: (i) peak search, (ii) indexing the pattern to determine the single orientation of the probed volume and (iii) determining the strain tensor. In the following the main steps are recalled.

\subsection{Peak search and indexing}

First, a background removal (detailed in the LaueTools software documentation available at https://sourceforge.net/ projects/lauetools/) followed by a peak search procedure are performed with different possible algorithms to determine the pixel locations of intensity maxima in the image. This is followed by a fit by a two-dimensional Gaussian to refine peak positions. Second, peaks are indexed by calculating the angles between all reciprocal-lattice vectors taken two by two within a selected subset of peaks and matching these angles to the theoretical ones for the considered crystal structure. In this way a single consistent crystal orientation matrix that indexes the highest number of Laue spots is determined. This method inherently reduces the initial two-dimensional data set into a one-dimensional one, since two angles are needed to express the direction of each diffraction vector (or equivalently two coordinates to express the peak position data on the detector area) but then only the angle between pairs of vectors (one parameter) is retained for the analysis, as shown in Fig. 1. By definition, the crystal orientation matrix is a rotation matrix that transforms the orthogonal sample reference frame to an orthogonal reference frame attached to the crystal lattice. Finally, an additional rotation is required to relate the crystal reference frame to a pre-defined reference frame attached to the instrument: here the $\mu$ Laue laboratory reference frame. Fig. 2(a) shows the used sample and $\mu$ Laue reference frames.

In the conventional LaueTools software, the positions of the reflections with respect to this reference frame are calculated by the following description of the reciprocal-lattice vector $\mathbf{q}_{h k l}$ :

$$
\mathbf{q}_{h k l}=\mathbf{U} \mathbf{B} \mathbf{G}_{h k l},
$$

where $\mathbf{G}_{h k l}$ is the reciprocal-lattice vector for the $h k l$ reflection, $\mathbf{G}_{h k l}=h \mathbf{a}^{*}+k \mathbf{b}^{*}+l \mathbf{c}^{*}(h, k, l$ are the Miller indices of the diffracting plane), expressed in an orthogonalized crystal 
reference frame established after Busing \& Levy (1967), when multiplied by B. The matrix $\mathbf{U} \mathbf{B}$ describes the mechanical transformation (i.e. deformation gradient tensor expressed in the reciprocal space), where $\mathbf{B}$ depends on the elastic strain tensor $\boldsymbol{\varepsilon}$ of the gauge volume being scanned by the X-ray beam, and $\mathbf{U}$ is a combination of rotations needed to express vector components in the laboratory reference frame indicated in Fig. 2. Since $\mathbf{a}^{*}, \mathbf{b}^{*}, \mathbf{c}^{*}$ only depend on the crystal structure, it is possible to obtain and refine the matrices $\mathbf{U}$ and B by using $h \mathrm{kl}$ reflections coming from (at least) four different planes that are not mutually parallel, yielding different $\mathbf{q}_{h k l}$ and $\mathbf{G}_{h k l}$ coordinate values (Chung \& Ice, 1999).

\subsection{Strain refinement}

The final part of the analysis is to refine the strain tensor (on which $\mathbf{B}$ depends) within the measured volume using the small shifts in peak positions, obtained by a Gaussian fit on the experimental peak positions, with respect to the theoretical positions given for the refined orientation matrix. The shifts can be as small as $0.005^{\circ}$, corresponding typically to a peak shift of a few tenths of a pixel (for a typical setup, i.e. specimen-detector distance of $\sim 60 \mathrm{~mm}$, pixel size of $\sim 80 \mu \mathrm{m}$ ). The refinement of the strain tensor is carried out with the LaueTools software, allowing the lattice parameters to deviate slightly from their theoretical values. This is done by minimizing the difference between measured directions of reciprocal-lattice vectors and theoretical directions. As the exact value of the wavelength for a given reflection is unknown in a Laue experiment, only a relative change in lattice parameters (shape change) can be calculated by such a method. This corresponds to the deviatoric strain tensor. Then, volume changes of the given unit cell, corresponding to the trace of the elastic strain tensor, are undetectable unless the X-ray energy of at least one Laue spot is measured (Robach et al., 2013).

\section{EBSD-assisted $\mu$ Laue approach}

As presented in $\S 1$, indexing becomes difficult when there are several crystals diffracting simultaneously in the $\mu$ Laue probed volume. One way to solve this problem is to introduce an a priori knowledge of the orientation of at least a subset of crystals in the polycrystalline volume being probed during the microdiffraction experiment. We propose to use the crystal orientation determined by EBSD to assist the indexing of $\mu$ Laue patterns. Employing a SEM-based technique is also helpful to decide on the locations of the ROI to be analysed, based on the microstructural features (a region of larger or finer grains, areas in the vicinity of a crack etc.).

\subsection{EBSD measurements}

For EBSD, the sample needs to be tilted by $70^{\circ}$ in the scanning electron microscope to reach the measurement condition; then the detector is inserted (Fig. 2b). The incident electron beam scans the ROI and at each beam position the Kikuchi diffraction pattern is automatically indexed to determine the crystal orientation with respect to the specimen reference frame. Classically, the resulting absolute orientation of each single crystal is determined with an accuracy of $1-2^{\circ}$ (Humphreys, 2001).

As the EBSD-assisted $\mu$ Laue technique relies on the complementary use of EBSD and $\mu$ Laue, one needs to be able to relate precisely the position of both EBSD and $\mu$ Laue scans, with an accuracy in the micrometre range. In order to achieve this, the sample was observed by SEM and the ROIs were marked by focused ion beam (FIB) milling. As the marker can be seen both with the scanning electron microscope and with the optical microscope available at the $\mu$ Laue beamline, it can be efficiently used to find the same ROI several times and match regions measured by EBSD and $\mu$ Laue with accuracy down to the micrometre range.

\subsection{Indexing by template matching}

Before the two-dimensional $\mu$ Laue patterns are processed, the crystal orientation determined by EBSD is expressed in the $\mu$ Laue reference frame, according to the conventions described in Fig. 2. After this frame conversion, a three-step refinement for the orientation matrix is carried out. The first two steps are described below, and the third is included in the standard strain refinement step.

For the same single crystal analysed by EBSD and $\mu$ Laue, the X-ray diffraction pattern simulated from the EBSD orientation only roughly matches the experimental $\mu$ Laue pattern. Because of the sample mounting, despite use of markers and careful sample alignment procedures, the orientation of the diffracting crystals cannot be exactly the same in the EBSD and $\mu$ Laue experiments. A typical difference in orientation determination of a few degrees is unavoidable. Part of this discrepancy is associated with the EBSD orientation accuracy, discussed above. To adjust the orientation determined by EBSD to the $\mu$ Laue one, we make use of a method labelled 'template matching': diffraction patterns are calculated for a large number of crystal orientations. The calculated patterns are then compared one by one with the experimental one, and the pattern matching best is retained. A procedure along the same lines was used by Rauch \& Dupuy (2005) to index transmission electron microscopy diffraction patterns. To correct the global misorientation between EBSD and $\mu$ Laue, template matching with a relatively large rotation step $\left(\sim 0.5^{\circ}\right)$ around each of the axes $x_{\mathrm{L}}, y_{\mathrm{L}}$ and $z_{\mathrm{L}}$ between calculated images is first applied. This procedure is carried out for all three axes over a range of about $\pm 4^{\circ}$ and the orientations that best match the experimental data are listed. From this best matching matrix, one can estimate the mean orientation of clusters of crystals slightly misoriented with respect to each other which generate Laue spots that are close to each other and possibly overlapped. This mean matrix is then taken as the initial crystal orientation for further orientation refinements. We make use of a second template matching with a smaller orientation step (typically $0.05^{\circ}$ ) in which we aim to disentangle the orientation of each crystal belonging to the cluster of crystals mentioned above. Doing so, we make sure to determine the precise orientation matrix of all small crystals 


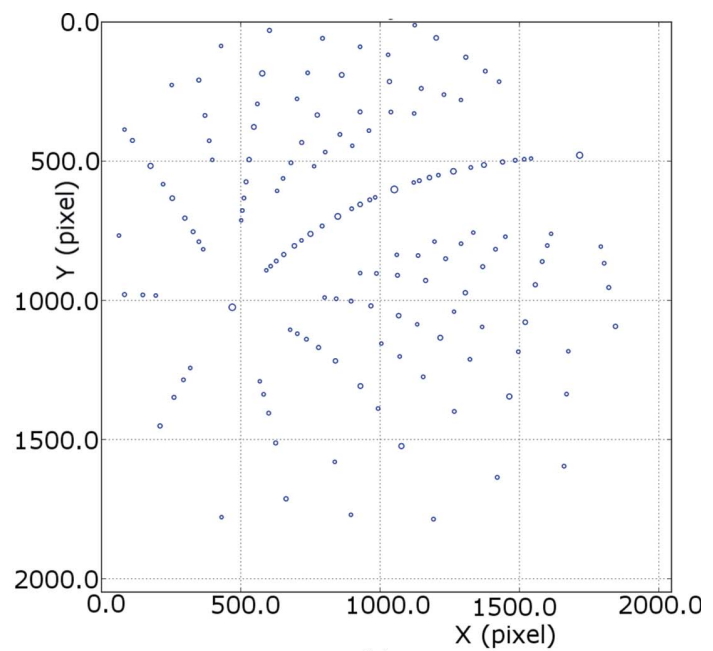

(a)

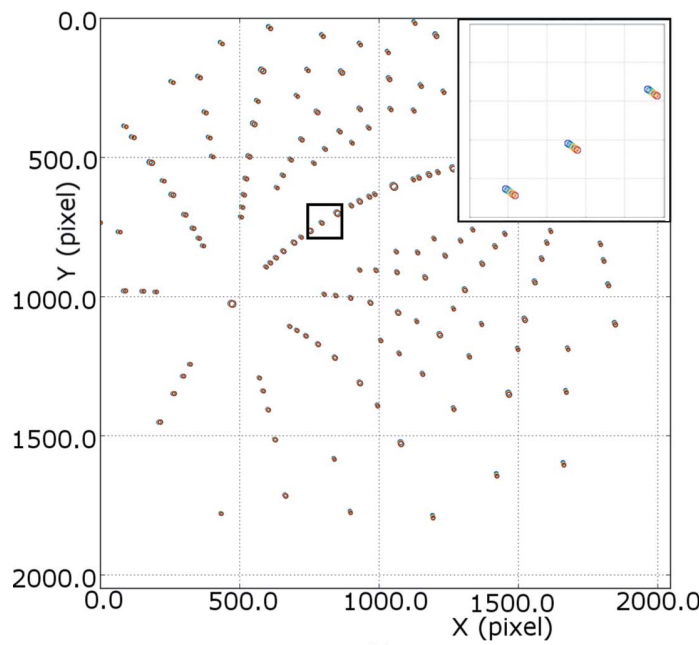

(b)

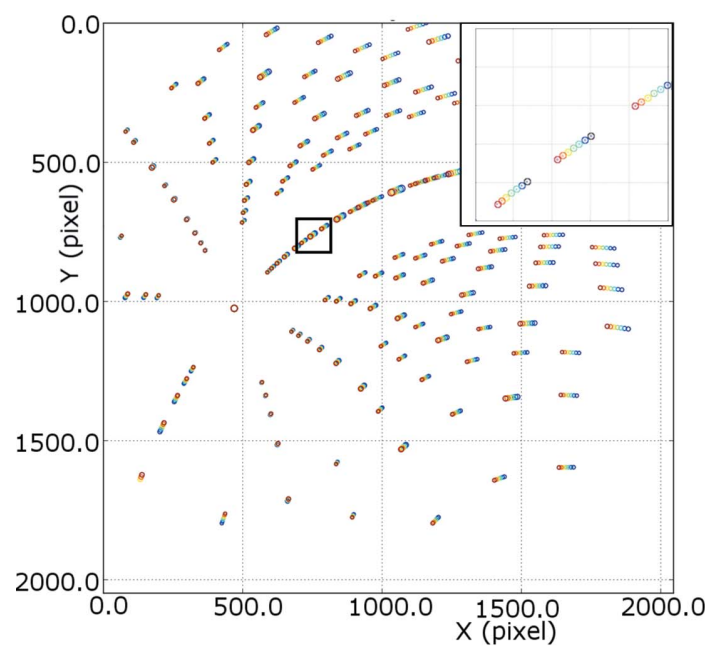

(c)

Figure 3

Simulated $\mu$ Laue patterns of $(a)$ a single crystal with a monoclinic structure in the $P 2_{1} / c$ space group; $(b)$ six crystals of the same phase with rotations of $\varphi$ around the (001) plane normal, where $\varphi=0,0.2,0.4,0.6,0.8$ and $1^{\circ}$, and detail of the marked region (inset); (c) seven crystals of the same phase with an applied strain of $-3,-2,-1,0,+1,+2$ and $+3 \%$ along the (001) plane normal, and detail of the marked region (inset). within the volume irradiated by the X-ray beam. To illustrate this, an example showing the effect of slight changes in the orientation matrix is given in Figs. 3( $a)$ and 3(b). As seen in the figures, the shifts in peak positions are relatively small in such a case, about one detector pixel for $0.2^{\circ}$ of rotation. $\mu$ Laue allows determination of the peak positions with a precision of 0.5 pixels or even better. After this second stage of orientation refinement, a small number of crystals with slightly different orientation matrices are selected from a single $\mu$ Laue pattern for the strain refinement step.

Finally, orientation and strain are refined at the same time for each crystal belonging to the cluster using the standard procedure. A small shift in the ideal positions of the Bragg reflections is expected due to strain in the crystal. Examples of the magnitudes of such shifts are visible in the calculated pattern given in Fig. 3, calculated considering a strain effect along the (001) plane normal.

\section{Application of the method to zirconia-based materials}

\subsection{Material}

As introduced above, an application to a zirconia-based material is now provided as an illustrative example of the proposed EBSD-assisted $\mu$ Laue method. The sample $(\sim 5 \times$ $5 \times 10 \mathrm{~mm})$ used for this experiment was cut from a large fused-cast block $(\sim 250 \mathrm{~kg})$ that was produced by the company Saint-Gobain CREE. It is made of sub-millimetre zones of pure monoclinic zirconia embedded in a silica-rich glassy phase (see Fig. 4). After cutting, the sample was carefully polished in order to be well suited to EBSD experiments.

During its casting from the melt, pure zirconia solidifies in a cubic $(F m \overline{3} m)$ structure at $2973 \mathrm{~K}$; then it transforms to tetragonal $\left(P 4_{2} / n m c\right)$ at $2573 \mathrm{~K}$ and finally to monoclinic $\left(P 2_{1} /\right.$ c) (see e.g. Smirnov et al., 2003). This last SPT takes place at around $1443 \mathrm{~K}$ under ambient pressure. It is usually associated with a volumetric expansion close to $4 \%$ (Kisi \& Howard, 1998). As a result of these successive SPTs, a multi-scale microstructure containing nano-sized monoclinic zirconia crystals is formed (see Fig. $4 b$ ). There are strict orientation relationships between the orientations of the initial parent cubic crystal and all the resulting monoclinic crystals observed at room temperature. Up to 24 monoclinic variants can theoretically be generated from the initial cubic crystal (Humbert et al., 2010).

As a result of its processing history and the SPTs, the material contains high amounts of internal residual stresses which usually generate cracks and can cause early failure (Cockcroft et al., 1994; Patapy et al., 2009; Örs et al., 2017). There are three main causes of internal stress fields in the specimen. The first is the lattice shearing and volume expansion during the SPTs. The second is related to the anisotropy of the coefficients of thermal expansion of the tetragonal and monoclinic zirconia (Frey et al., 1990). The third main cause, which is associated with the specific processing conditions, is inhomogeneous temperature distribution during casting. These three effects are clearly correlated with each other and 
induce strains at different scales. EBSD-assisted Laue microdiffraction experiments address the determination of strains at the scale of the coherently diffracting crystalline domains.

\subsection{Experimental result: EBSD and $\mu$ Laue data acquisition}

A Zeiss AURIGA FIB scanning electron microscope was used to localize the ROI [see the $45 \times 65 \mu \mathrm{m}$ rectangle in

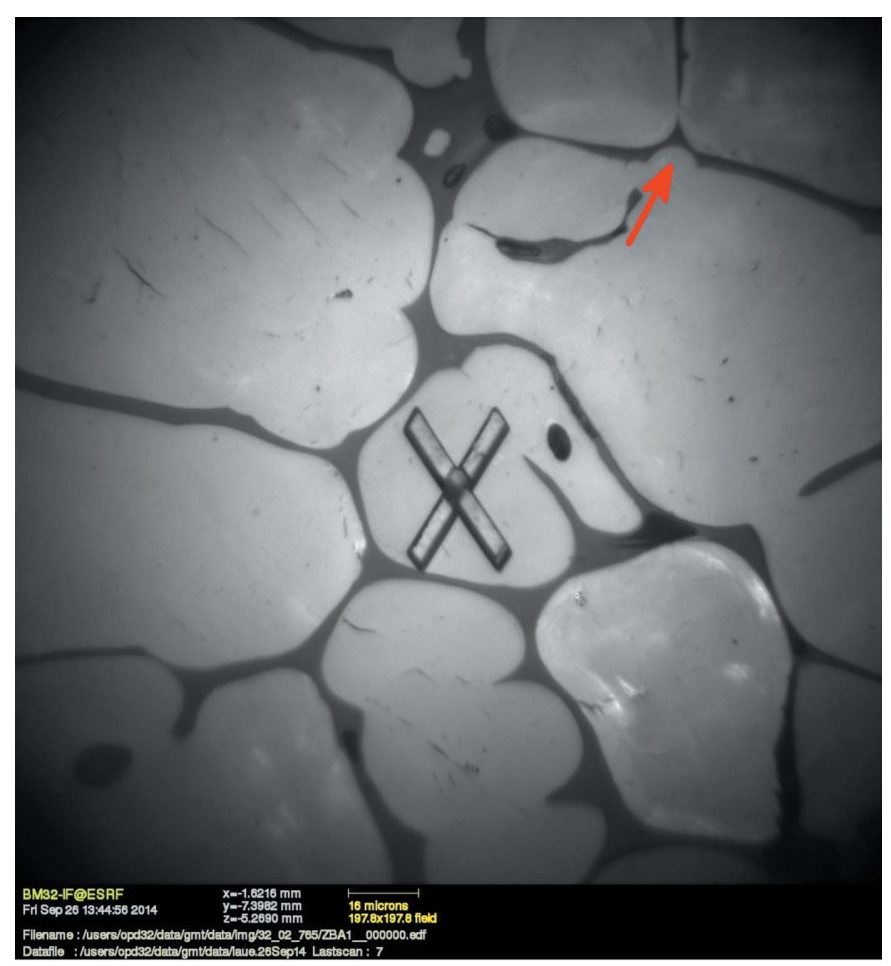

(a)

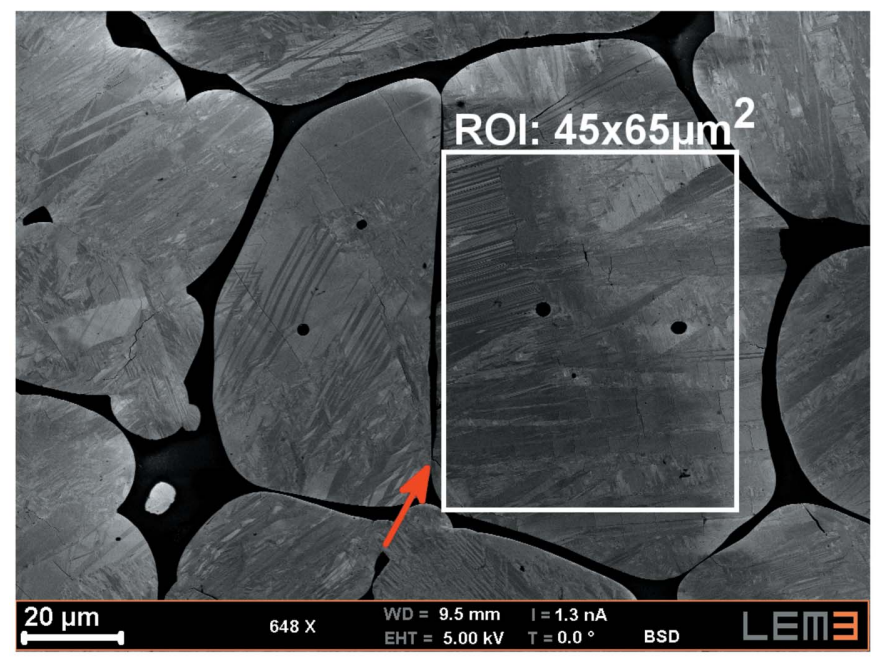

(b)

\section{Figure 4}

Microstructure of the sample. (a) $\mathrm{ZrO}_{2}$ areas (grey shades) in a glassy matrix (black) and (b) close-up of a $\mathrm{ZrO}_{2}$ area, showing the needle- and plate-like monoclinic $\mathrm{ZrO}_{2}$ crystals. The cross-shaped marker in $(a)$ produced by FIB milling is used to find the same ROI during the $\mu$ Laue and EBSD experiments (the red arrow marks the same microstructural feature in both figures).
Fig. 4(b)] and to mill a nearby cross-shaped marker (Fig. 4a). The backscattering electron (BSE) image in Fig. 4(b) shows the microstructure of the zirconia-based material; the glassy phase (in black) surrounds a dendrite skeleton solidified in the cubic phase and fully transformed to monoclinic with the presence of micro-cracks. The orientation contrast in BSE mode reveals the nano-sized monoclinic domains with twins. More details on this specific microstructure are given by Patapy et al. (2013).

EBSD measurements were realized in a Zeiss SUPRA 40 scanning electron microscope equipped with Bruker QUANTAX hardware and ESPRIT software. An accelerating voltage of $15 \mathrm{kV}$ and a working distance of $\sim 15 \mathrm{~mm}$ were used throughout the analysis. The step size for the EBSD scan was set at $0.2 \mu \mathrm{m}$ in order to capture the orientations of the larger monoclinic domains. The orientation data were treated with the EBSD software as well as in-house-developed scripts.

Fig. 5 gives the EBSD result by means of the inverse pole figure (IPF) colour map. The standard IPF and its colour key are given in the inset. In this work we concentrate on the righthand side of the EBSD map, which corresponds to the ROI marked in Fig. 4(b). This ROI covers a single parent cubic crystal as shown by the experimental pole figures in Fig. 6(a) (see also Patapy et al., 2013). The parent cubic phase orientation was calculated according to a method described earlier (Humbert et al., 2010) (Fig. 6b), as were the orientations of the 24 theoretically possible monoclinic variants (Fig. $6 c$ ). These simulated orientations are very similar to the measured ones, indicating that the 24 variants are present in the ROI. However, because the successive SPTs occur during cooling, the experimental orientations are spread $\sim 1-2^{\circ}$ around the simulated variants. Accordingly there is a strong microtexture within the ROI.

Laue microdiffraction experiments were carried out at the French CRG beamline BM32 at the European Synchrotron

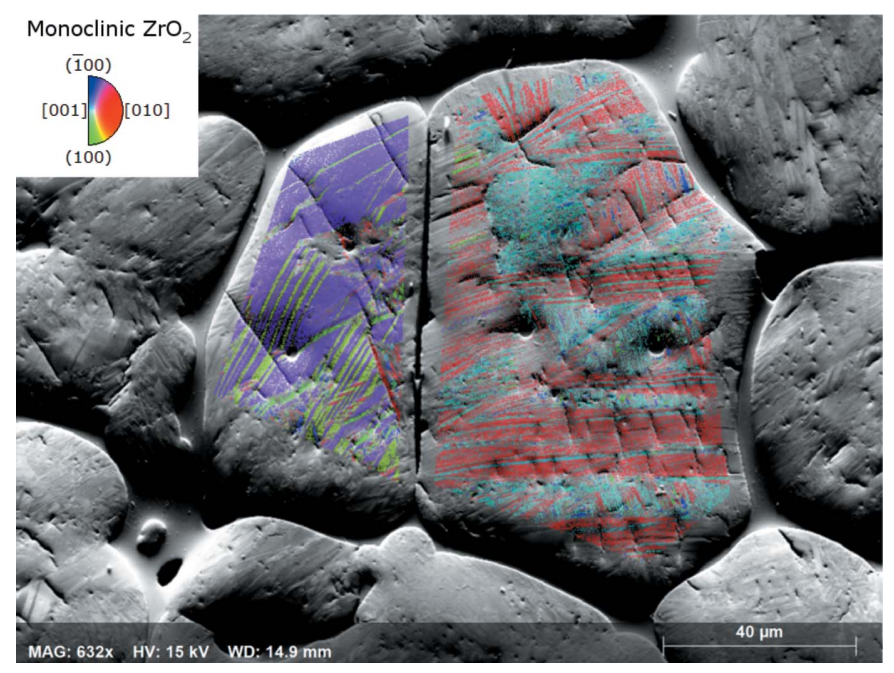

Figure 5

Inverse pole figure map along the $z_{\mathrm{E}}$ direction, measured by EBSD. It covers a region belonging to the ROI shown in Fig. $4(b)$. The colour code indicating the crystal orientation is given in the inset. Backscattered electrons have been used to obtain the grey background image. 
(ESRF, Grenoble, France). The ROI previously analysed by EBSD was further scanned with $\mu$ Laue with a step size of $1 \mu \mathrm{m}$. A polychromatic beam $(5<E<22 \mathrm{keV})$ was used. The beam was focused down to $\sim 300 \times 300 \mathrm{~nm}$ in cross section (at the focal point) by Kirkpatrick-Baez mirrors (Ulrich et al., 2011). The sample stage was inclined by $40^{\circ}$ with respect to the incoming X-ray beam. The vertical distance between the detector plane and the point on the sample surface where diffraction occurs was $\sim 70 \mathrm{~mm}$. A MAR CCD165 (now Rayonix) detector $(79.14 \mu \mathrm{m}$ pixel size, $2048 \times 2048$ pixels, 16 bit dynamic) was used to record the diffraction patterns. To measure the direction of the diffracted beam accurately, it is necessary to calibrate the geometrical parameters, i.e. the orientation of the incident X-ray beam and the position of the probed zone with respect to the detector. A strain-free Ge single-crystal wafer was mounted close to the measured sample and was used for this purpose. Details of the setup geometry and calibration procedure are discussed by Ulrich $e t$ al. (2011). The data obtained by microdiffraction experiments are treated by the software LaueTools which is presented, for example, by Robach et al. (2017).

Fig. 7 shows an example of a typical $\mu$ Laue diffraction pattern of a zirconia region within the mentioned ROI. For the reasons detailed earlier, the pattern is too complex to be analysed with the conventional indexing routines. Tremendous computation time would be necessary, with a result exhibiting a significant probability of wrong peak assignment. We will
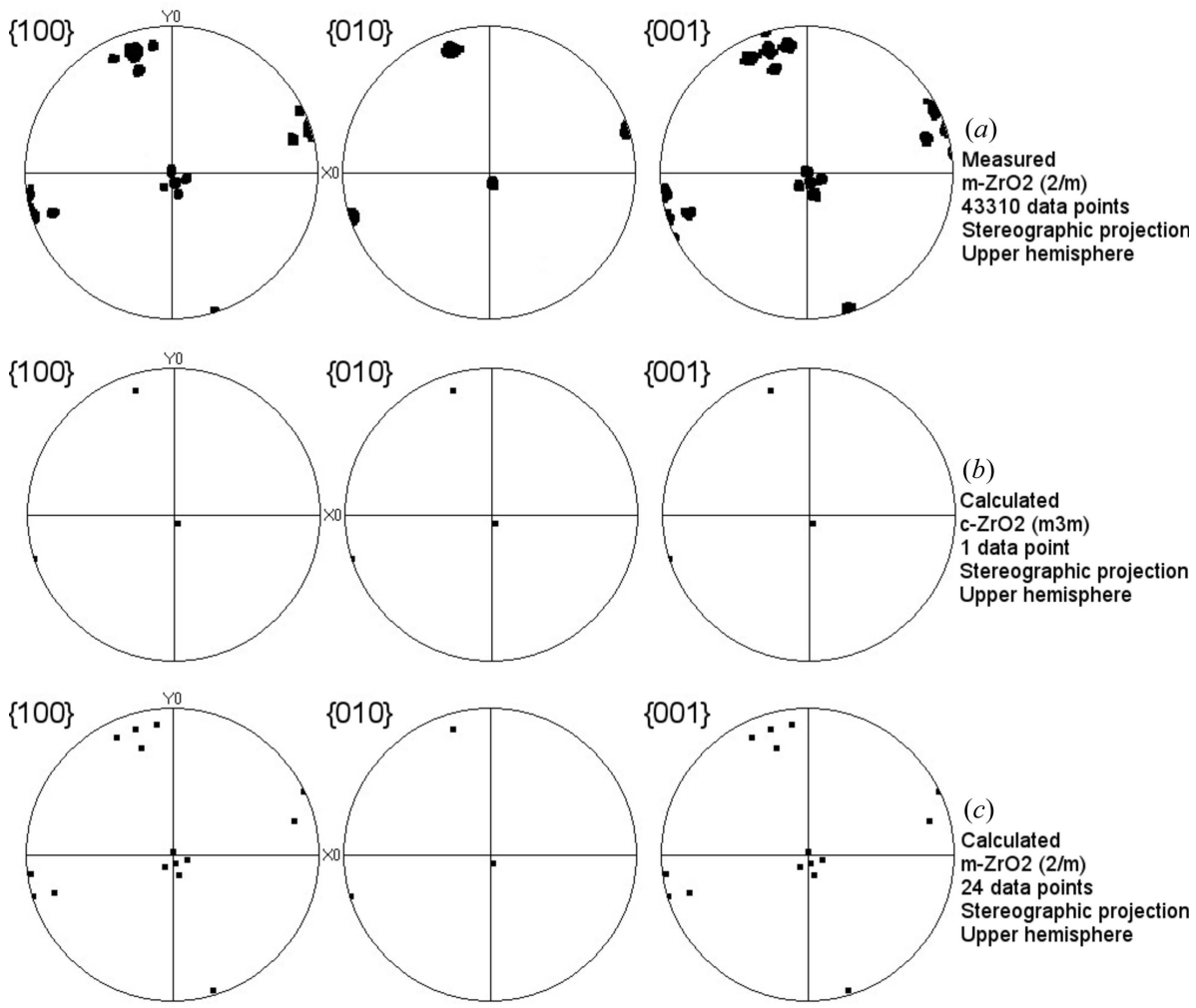

Figure 6

Crystal orientation analyses of the ROI in Fig. 4(b), as provided by EBSD. (a) Experimental pole figures of the monoclinic $\mathrm{ZrO}_{2}$ phase, $(b)$ calculated average orientation of the cubic parent crystal of the ROI, and (c) calculated orientations of the 24 monoclinic variants resulting from this cubic parent. show in the following how the proposed EBSD-assisted $\mu$ Laue approach allows extraction of quantitative strain information from such measurements.

\subsection{EBSD-assisted $\mu$ Laue data treatment}

To treat the $\mu$ Laue digital images, firstly an efficient peak search procedure is required. One needs as many experimentally measured reflections as possible to be able to select the largest number of spots from each crystal contribution. Therefore a search to obtain all the local maxima above a certain threshold (250 pixel counts, where the pattern maximum was 34745 counts) in a given box of $5 \times 5$ pixels was carried out, which yielded about 700 peaks. Precise positions of these peaks were obtained by fitting the same $5 \times 5$ pixel box with a two-dimensional Gaussian function.

Afterwards, orientation input from EBSD of the given scan point is entered into the program either as the crystal orientation matrix measured directly for this point or as the orientation of one of the matching 24 variants as calculated from the parent cubic orientation earlier. In both cases, this guessed orientation matrix gives simulated peak positions slightly shifted from the measured ones for reasons explained in §3.2. Thus the template matching algorithm is used to refine the EBSD measured orientation to match the mean cluster peak positions in the $\mu$ Laue pattern. Fig. 8 shows the initial pattern simulated with the orientation from EBSD and the best matched pattern after this first template matching step. The crystal rotation screening range needed to achieve this match is typically $3-4^{\circ}$.

Here, we have taken advantage of the microtexture of the specimen, which allows us to calculate in advance the orientations of the 24 variants present in the diffracting volume. The advantage of this microtexture is that it provides an opportunity to make the treatment procedure automatic without the need for synchronizing very precisely the positions of scan points of EBSD and $\mu$ Laue data. For specimens without a microtexture, such as most materials that have not experienced SPT, assistance of $\mu$ Laue from EBSD would basically work the same way, but in that case one needs to define precisely which pixel of the EBSD map corresponds to the actual $\mu$ Laue pattern.

For each $\mu$ Laue pattern, the probed volume consists of several sub-micrometre-sized crystals slightly disoriented from each other but belonging to the same variant. This is clearly shown in Fig. 9, where the $\mu$ Laue pattern corresponds to two or three sets of conics. Each diffraction spot corresponding to 
the same $(h k l)$ lattice planes of a given variant is split into several components and, as shown in Fig. 9(b), some of them appear to be elongated.

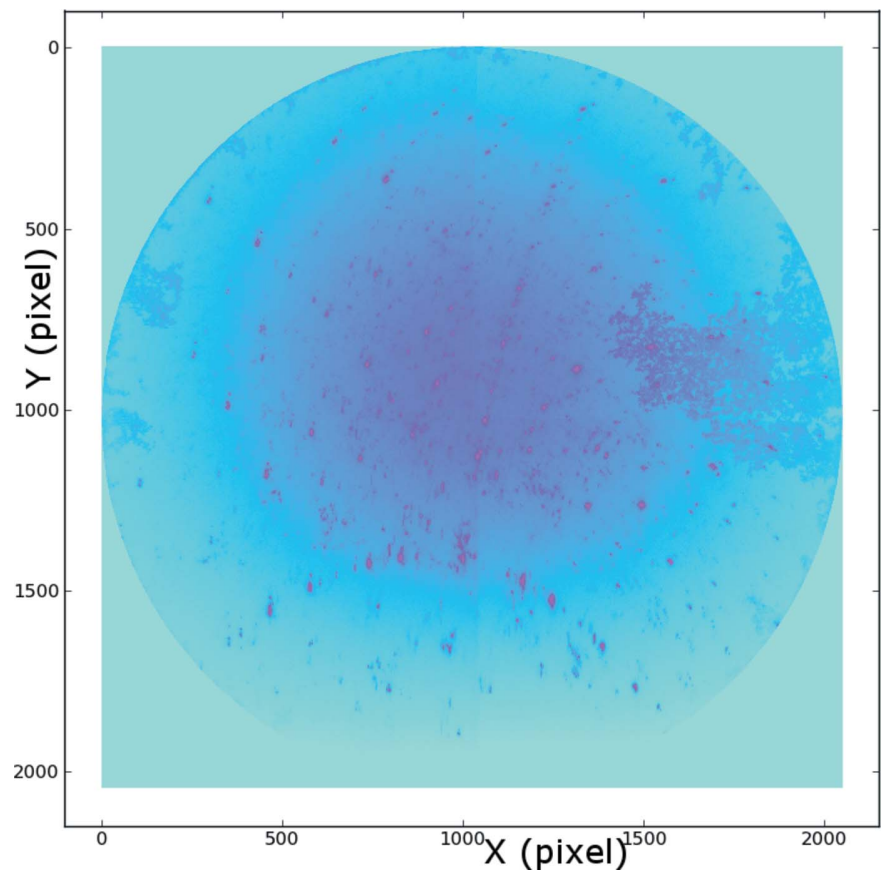

Figure 7

$\mu$ Laue diffraction pattern of the sample, showing peaks from numerous $\mathrm{ZrO}_{2}$ crystals. The splitting of a number of diffraction spots is due to the contribution of many different small crystals having slightly different orientations (within less than $0.5^{\circ}$ of one another). A close-up is shown in Fig. 9.

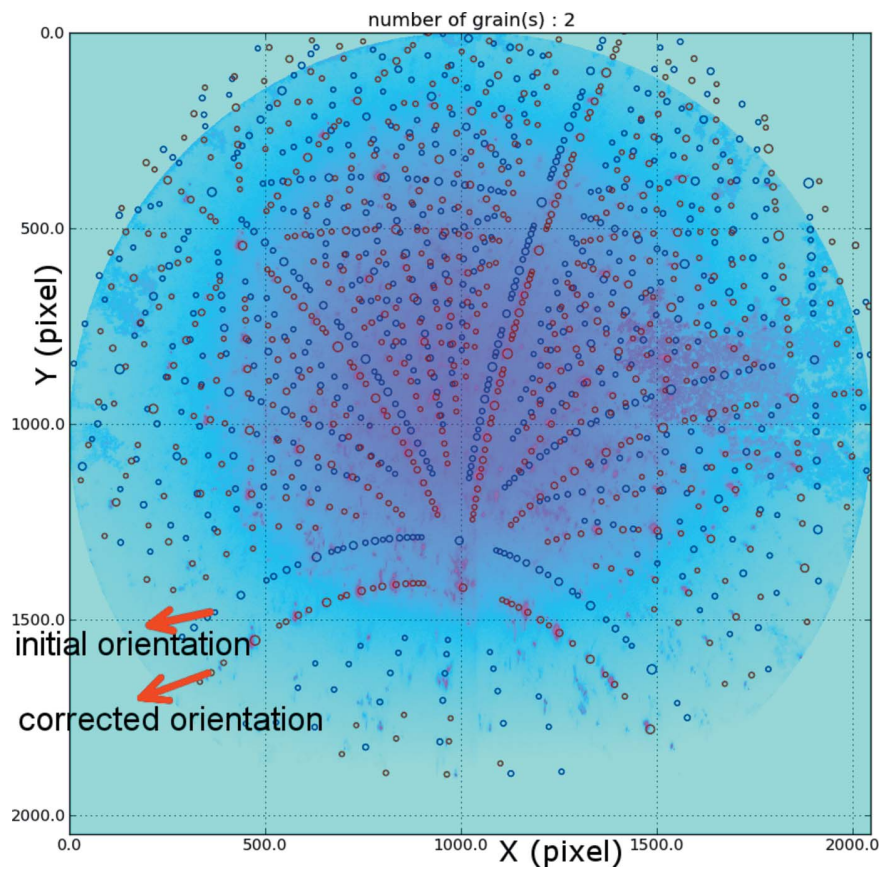

Figure 8

The optimization of orientation by 'template matching'. Crystal orientations after EBSD calculation (in blue, labelled 'initial orientation') and after template matching (in red, labelled 'corrected orientation') over the same diffraction pattern shown in Fig. 7.

\subsection{Microstrain measurements}

After determining the orientation of individual crystals, we performed refinement for strain and orientation for each of them separately. Positions of the peaks corresponding to the strain-free monoclinic crystal were obtained from the lattice parameters published earlier (Howard et al., 1988). A fine angular tolerance value was chosen to ensure the selection of peaks that correspond unambiguously to the same $\mu$ Laue pattern spot set, avoiding detrimental misassociation between experimental and simulated spots.

The resulting component values of the deviatoric strain tensor obtained by the refinement of the $\mu$ Laue pattern shown in Fig. 7 are between 0.01 and $-0.5 \%$ depending on the strain component being analysed. For more than 50 different analysed diffraction patterns, the values are in the range of $\pm 3 \%$ for $\varepsilon_{33}$ and $\pm 1 \%$ for $\varepsilon_{11}$ and $\varepsilon_{22}$. Depending on the crystal and the scan position on the sample, a significant shear strain component can also be observed.

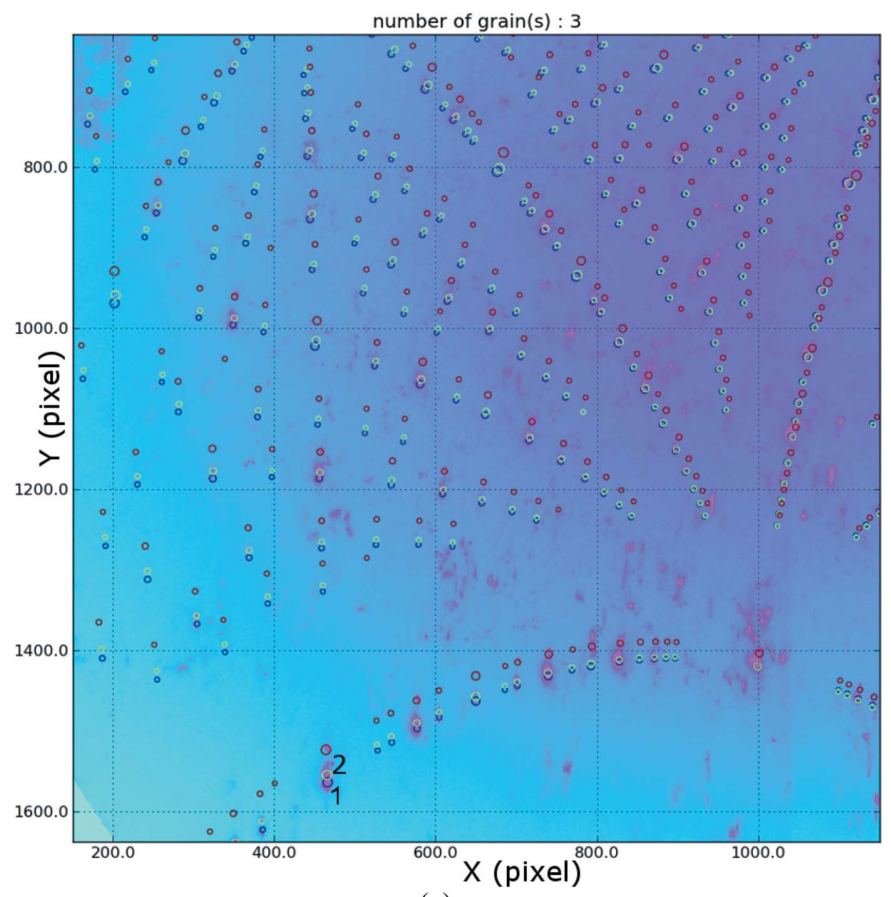

(a)
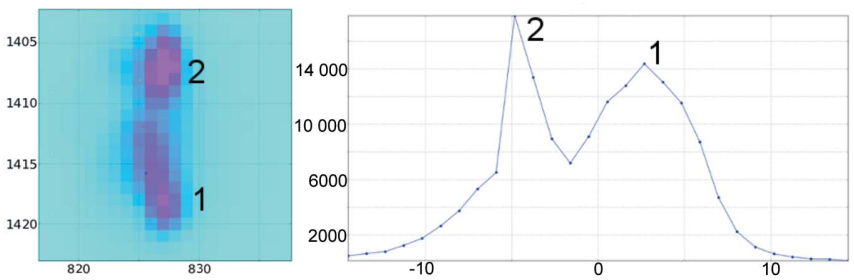

(b)

Figure 9

(a) Results of the second template matching stage in finer angular steps. Only a part of the diffraction pattern is shown here. At least three different crystals with small differences in orientation can be detected, marked by different colours. (b) Close-up of the region marked by 1 and 2 in $(a)$, showing the clear spot splitting on the two-dimensional detector image as well as the intensity profile (one-dimensional section) around the peaks along the $y$ direction. 


\section{Discussion}

The developed approach allows determination of strains in an accurate and efficient way in a sample composed of submicrometre crystals of low symmetry, with the possibility of discriminating slightly disoriented crystals. In the following we will discuss the reliability and reproducibility of the obtained results. A short physical explanation of the strains measured in zirconia crystals will also be given, although the micromechanical aspects are beyond the scope of this paper and will be presented elsewhere.

It should be noted that the resulting strain tensor is for a given crystal orientation observed in a particular diffraction pattern generated by the probed volume. Therefore, if there are in this volume two different crystals that have exactly the same orientation matrix, the tensor obtained will be the average deformation for the two crystals.

\subsection{Reliability of the method and potential pitfalls}

A useful indicator for the quality of the strain refinement is the mean value of the deviations between the experimental peak positions obtained by Gaussian fit and the best adjusted one obtained after strain refinement. For an acceptable refinement, this value should be below 1 pixel. Mean deviations of our data are usually of the order of 0.3 pixels over typically 100 spots. As mentioned, values of strain in the range of $\pm 3 \%$ are obtained for our application example. The fact that the mean pixel deviations are always lower than 1 pixel shows that the desired precision is achieved. Nonetheless, to confirm the results we need to examine potential issues that could affect the calculations.

The principle of refinement is to minimize the cost function expressed by the sum of distances between experimental and calculated spot positions $\left(\chi=\sum_{i}\left\|\mathbf{r}_{\text {exp }}^{i}-\mathbf{r}_{\text {calc }}^{i}\right\|\right)$. The most important potential source of error would be to include wrong peak assignation in the cost function, i.e. to include a measured peak coming from a different crystal. Using the proposed EBSD-assisted $\mu$ Laue approach, this has been avoided.

The refined strain values are robust against differently chosen peak subsets belonging to a given crystal. To ensure this, we made a strain refinement on the same $\mu$ Laue pattern with different peak lists. Firstly, a full list of 120 peaks found to belong to the same crystal after refinements was isolated in a diffraction pattern (a different pattern than above). Then, 100 different subsets containing 80 randomly chosen peaks each are generated from this principal list of 120 peaks. Refinements were done with exactly the same parameters (detector calibration parameters, initial orientation matrix and angular tolerance) for all the subsets. From the initial set of 80 peaks, different numbers of peaks could be indexed, and these were subsequently used for final strain refinement. Fig. 10 shows the values for all components of the deviatoric strain as a histogram plot obtained for a single crystal orientation with this procedure. The histogram shows little spread in values. The small standard deviation of strain values (the largest deviation is observed along the $\varepsilon_{33}$ direction, $0.05 \%$ ) confirms the relatively narrow distribution. The mean value obtained again by
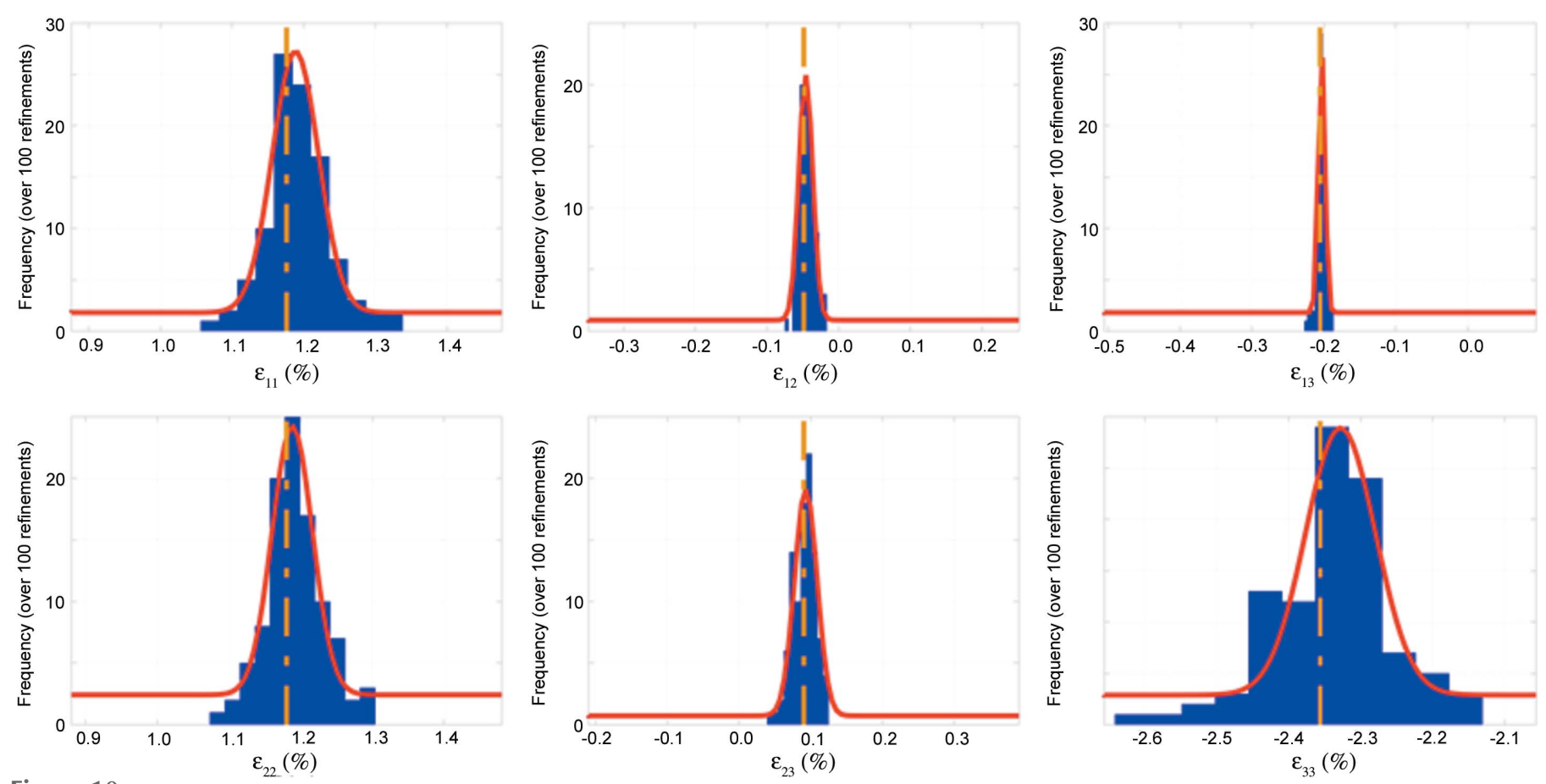

Figure 10

Histogram of all the strain components for a single crystal orientation on a single $\mu$ Laue pattern as calculated by 100 randomly chosen sets of 80 peaks. The Gaussian fits are shown by the red curves, while the vertical lines correspond to the strain values yielded by the refinement of the whole list of 120 peaks. These values compare well with the maxima of the Gaussian function, while the standard deviation indicates a narrow distribution and fairly reproducible strain values. 
the Gaussian fits compares well with the value obtained by refining the full set of 120 peaks (again, for $\varepsilon_{33},-2.33$ and $-2.36 \%$, respectively; the differences for the other directions are smaller). These results show that the initial peak list does not contain a significant number of reflections from a second crystal with a different state of deformation. This analysis was made for other strain components and different diffraction patterns, and the results were very similar. It has also been verified that the choice of the angular tolerance parameter (generally between 0.01 and $0.05^{\circ}$ ) did not have a significant effect on the results presented.

After careful visual analyses of many diffraction patterns, all the peaks that lie close to each other, indexed and associated with slightly different crystal orientations, were found to be on different experimentally measured peak maxima. Accordingly it is ensured that the indexing procedure runs correctly without 'cross-assignment' of two different crystals as one.

The deviatoric strain $\varepsilon_{33}$ was very often the largest observed component compared with the other ones. The fact that we observe highest strain values in that direction hints towards a physically plausible phenomenon. That is, internal stresses develop along the $\langle 001\rangle$ or (001) plane normal direction in a single crystal (single orientation matrix) of monoclinic $\mathrm{ZrO}_{2}$ upon cooling. Such a result is consistent with the anisotropy of the thermal expansion coefficient. Micro-cracks perpendicular to this direction and due to stress release are very common in heat-treated samples of the same material (Sibil et al., 2011).

\subsection{Micromechanical explanation of the observed strain values}

The points discussed above indicate that the EBSD-assisted $\mu$ Laue method provides reliable strain level assessments. For the physical explanation of the results obtained one should look into the details of the manufacturing process of such materials. As discussed earlier, depending on the initial stress field, SPTs occur at different stages of cooling in different parts of the block. The final tetragonal-to-monoclinic SPT implies a few per cent of change in unit-cell volume, which creates large stresses in the considered area corresponding to one initial cubic crystal. Together with the anisotropy of the thermal contraction of monoclinic zirconia (Frey et al., 1990), a large stress concentration is expected during cooling, leading to either cracks or large elastic strain.

In agreement with these arguments, strain values up to $0.1 \%$ based on neutron diffraction results with much larger gauge volumes (millimetre size) than in the present work were reported recently (Örs et al., 2017). Because of the size of the diffraction volume, strains observed with that method are averaged over a very large number of crystals. Much higher values are expected from microstrain measurement at smaller scales in individual crystals.

Besides the measured elastic strain, there are other phenomena observed on the $\mu$ Laue diffraction patterns (peak splitting, neighbouring crystals with very small orientation differences and the diffuse intensity between the Laue reflections) that hint at mechanisms of stress accommodation. The detailed micromechanical analysis of the results and its implications for zirconia-based materials will be the subject of a separate paper.

\subsection{Advantages of EBSD-assisted $\mu$ Laue}

The EBSD-assisted $\mu$ Laue method, which relies on the input of prior knowledge of an approximate crystal orientation followed by an orientation screening procedure, opens up new possibilities for $\mu$ Laue analysis. Complex materials with fine microstructure and low crystal symmetry can be analysed with the help of this new approach, and $\mu$ Laue analysis will become much less microstructure dependent. This applies similarly to $\mu$ Laue patterns acquired at high X-ray energies as they are also highly populated with many spots. Even in cases of polycrystalline $\mu$ Laue patterns where conventional indexing is possible, EBSD-assisted $\mu$ Laue possesses numerous advantages over the conventional routine. Firstly, the accuracy of the measured strain values is improved. In the absence of any prior orientation data, one has to single out peaks from the pattern that are likely to come from a single crystal. Since this is a highly arbitrary process, the correctness of the pair association obtained would always be in question. In our method we consider all the measured peaks on the pattern and select those that belong to a single orientation. Subsequently, the results are much more reproducible, as shown before. Time efficiency is increased quite remarkably as well: the treatment of a single pattern by a careful manual selection of peaks is an iterative 'trial-and-error' process for complex diffraction patterns; it usually takes up to 4-5 h to get through a single pattern.

With EBSD-assisted $\mu$ Laue it is a matter of minutes on a standard computer to analyse a single pattern from peak search to the strain refinement. Calculation of the orientation of the cubic phase and of the resulting 24 variants from EBSD, and subsequent reference frame change, are done for the whole sample ROI, taking of the order of $10 \mathrm{~min}$ for 2500 patterns. Afterwards, initial peak search and the subsequent orientation refinements with template matching take $\sim 6$ and $\sim 8$ min per $\mu$ Laue pattern, respectively. The ultimate orientation and strain refinement of a single crystal is usually a quick step lasting only $\sim 15 \mathrm{~s}$. Therefore the whole process is estimated to be $\sim 15$ min per pattern. The computation time can probably be further improved by optimizing the used software, using more powerful processors and precalculating template $\mu$ Laue patterns stored in a binary format, as in the study by Rauch \& Dupuy (2005). Exploiting this new efficiency, determination of strain maps for such polycrystalline samples exhibiting low crystalline symmetry becomes possible.

Furthermore, the proposed method also allows one to sort out the crystal lying at the specimen surface, among all crystals producing Laue spots on the pattern, as its orientation is known from the EBSD. This is of significant interest since $\mu$ Laue data can be enriched with many other laboratory techniques currently available such as SEM, EBSD, atomic force microscopy, profilometry etc., which allows one to 
characterize the specimen surface only. In this way, the deformation characteristics and the local behaviour of the material can be determined by coupling microstructural, stress and strain fields measured on the specimen surface (Plancher et al., 2017).

\section{Conclusion}

An EBSD-assisted $\mu$ Laue method is presented for the strain analysis of materials with low crystal symmetry and submicrometre microstructure. The indexing ambiguity encountered for the data treatment of $\mu$ Laue patterns recorded on such samples is solved by introducing the crystal orientation data measured by EBSD into the $\mu$ Laue treatment routine. This orientation is used as a guess for the indexing screening technique exploring an optimized and narrow angular range. In this way, one can benefit from the high spatial resolution provided by the EBSD to differentiate between different crystal orientations as well as the high angular resolution of $\mu$ Laue to measure strain at the micrometre scale with a resolution of $10^{-4}$ or even better. The template matching indexing method makes use of the two-dimensional distribution of Laue spots on the detector screen, as opposed to the conventional method which operates on a one-dimensional distribution of angles. This helps in retaining the full twodimensional information on the measured patterns during the data treatment and disentangling intricate $\mu$ Laue patterns.

A $\mathrm{ZrO}_{2}$-based refractory material was chosen as a sample case to test this approach. This material exhibits a multi-scale, micro-twinned microstructure and has low crystal symmetry. Large internal stresses are developed in this material during its manufacture and as a result of the SPTs. The analysis yielded strains between $\pm 3 \%$ for different crystal directions, which is generally in accordance with the expectations based on earlier studies.

The obtained results are shown to be stable and consistent with respect to the different peak identification strategies applied to the $\mu$ Laue diffraction patterns. The final angular resolution can be as high as $0.02^{\circ}$ with a mean distance of about 0.3 pixel on the detector screen between the modelled peak positions and the recorded ones (over more than 100 peaks). Data treatment duration and the accuracy of the results are greatly improved by our approach.

Generalization of EBSD-assisted $\mu$ Laue is possible for other materials with challenging microstructures. Materials where the effect of SPTs dominates the microstructure could be good candidates for future work. EBSD-assisted $\mu$ Laue paves the way for the indexing of complex-shaped peaks containing multiple components and the determination of strain in materials exhibiting sub-micrometre microstructure made of crystals characterized by low symmetry.

\section{Acknowledgements}

The authors thank Isabelle Cabodi and Olivier Bories (SaintGobain CREE) for the supply of the zirconia material, Fanny
Gouraud and Marc Huger (SPCTS) for help with sample preparation, and Michel Humbert and Alain Hazotte (LEM3) for the fruitful discussions. Moreover, the authors thank Odile Robach for discussions regarding the software for converting the EBSD files into LaueTools orientation matrices.

\section{Funding information}

This research was funded by the French National Research Agency under the project name 'ASZTech' (ANR-12-RMNP0007).

\section{References}

Borbély, A., Renversade, L. \& Kenesei, P. (2014). J. Appl. Cryst. 47, 1585-1595.

Boulle, A., Guinebretière, R. \& Dauger, A. (2005). J. Phys. D Appl. Phys. 38, 3907-3920.

Boulle, A., Masson, O., Guinebretière, R. \& Dauger, A. (2003). J. Appl. Cryst. 36, 1424-1431.

Bouscaud, D., Morawiec, A., Pesci, R., Berveiller, S. \& Patoor, E. (2014). J. Appl. Cryst. 47, 1699-1707.

Busing, W. R. \& Levy, H. A. (1967). Acta Cryst. 22, 457-464.

Chen, X., Dejoie, C., Jiang, T., Ku, C. S. \& Tamura, N. (2016). MRS Bull. 41, 445-453.

Chung, J.-S. \& Ice, G. (1999). J. Appl. Phys. 86, 5249-5255.

Clark, J. N., Ihli, J., Schenk, A. S., Kim, Y.-Y., Kulak, A. N., Campbell, J. M., Nisbet, G., Meldrum, F. C. \& Robinson, I. K. (2015). Nat. Mater. 14, 780-784.

Cockcroft, S. L., Brimacornbe, J. K., Walrod, D. G. \& Myles, T. A. (1994). J. Am. Ceram. Soc. 77, 1512-1521.

Dingley, D. J. (1975). Proceedings of the 8th Annual SEM Symposium, edited by O. Johari \& I. Corvin, pp. 173-180. Chicago: IIT Research Institute.

Frey, F., Boysen, H. \& Vogt, T. (1990). Acta Cryst. B46, 724-730.

Godard, P., Carbone, G., Allain, M., Mastropietro, F., Chen, G., Capello, L., Diaz, A., Metzger, T. H., Stangl, J. \& Chamard, V. (2011). Nat. Commun. 2, 568.

Hofmann, F., Abbey, B., Liu, W., Xu, R., Usher, B. F., Balaur, E. \& Liu, Y. (2013). Nat. Commun. 4, 2774.

Hofmann, F. \& Korsunsky, A. M. (2014). Strain and Dislocation Gradients from Diffraction, edited by R. I. Barabash \& G. E. Ice, pp. 82-124. Imperial College Press/World Scientific Publishing.

Howard, C. J., Hill, R. J. \& Reichert, B. E. (1988). Acta Cryst. B44, 116-120.

Humbert, M., Gey, N., Patapy, C., Joussein, E., Huger, M., Guinebretière, R., Chotard, T. \& Hazotte, A. (2010). Scr. Mater. 63, 411-414.

Humphreys, F. J. (2001). J. Mater. Sci. 36, 3833-3854.

Hÿtch, M. J., Putaux, J.-L. \& Pénisson, J.-M. (2003). Nature, 423, 270 273.

Jiang, J., Benjamin Britton, T. \& Wilkinson, A. J. (2015). Acta Mater. 94, 193-204.

Kacher, J., Landon, C., Adams, B. L. \& Fullwood, D. (2009). Ultramicroscopy, 109, 1148-1156.

Kirchlechner, C., Keckes, J., Micha, J. S. \& Dehm, G. (2011). Adv. Eng. Mater. 13, 837-844.

Kirchlechner, C., Keckes, J., Motz, C., Grosinger, W., Kapp, M. W., Micha, J. S., Ulrich, O. \& Dehm, G. (2011). Acta Mater. 59, 56185626.

Kisi, E. H. \& Howard, C. J. (1998). Zirconia Engineering Ceramics: Old Challenges - New Ideas, edited by E. H. Kisi, pp. 1-36. Zurich, Switzerland: Trans Tech Publications.

Larson, B. C., Yang, W., Ice, G. E., Budai, J. D. \& Tischler, J. Z. (2002). Nature, 415, 887-890.

Larson, B. C., Yang, W., Tischler, J. Z., Ice, G. E., Budai, J. D., Liu, W. \& Weiland, H. (2004). Int. J. Plast. 20, 543-560. 
Liu, W. \& Ice, G. E. (2014). Strain and Dislocation Gradients from Diffraction, edited by R. I. Barabash \& G. E. Ice, pp. 53-81. Imperial College Press/World Scientific Publishing.

Liu, W., Ice, G. E., Larson, B. C., Yang, W., Tischler, J. Z. \& Budai, J. D. (2004). Metall. Mater. Trans. A, 35, 1963-1967.

MacDowell, A. A., Celestre, R. S., Tamura, N., Spolenak, R., Valek, B., Brown, W. L., Bravman, J. C., Padmore, H., Batterman, B. W. \& Patel, J. R. (2001). Nucl. Instrum. Methods Phys. Res. A, 467-468, 936-943.

Marijon, J. B. (2017). PhD thesis, Arts and Métiers ParisTech, Paris, France.

Maurice, C., Driver, J. H. \& Fortunier, R. (2012). Ultramicroscopy, 113, 171-181.

Maurice, C., Fortunier, R., Driver, J., Day, A., Mingard, K. \& Meaden, G. (2010). Ultramicroscopy, 110, 761-762.

Oddershede, J., Schmidt, S., Poulsen, H. F., Sørensen, H. O., Wright, J. \& Reimers, W. (2010). J. Appl. Cryst. 43, 539-549.

Ohashi, T., Barabash, R. I., Pang, J. W. L., Ice, G. E. \& Barabash, O. M. (2009). Int. J. Plast. 25, 920-941.

Örs, T., Gouraud, F., Guinebretière, R., Huger, M., Michel, V. \& Castelnau, O. (2017). J. Eur. Ceram. Soc. pp. 2295-2302.

Pantleon, W., Wejdemann, C., Jakobsen, B., Poulsen, H. F. \& Lienert, U. (2014). Strain and Dislocation Gradients from Diffraction, edited by R. I. Barabash \& G. E. Ice, pp. 322-357. Imperial College Press/ World Scientific Publishing.

Patapy, C., Gault, C., Huger, M. \& Chotard, T. (2009). J. Eur. Ceram. Soc. 29, 3355-3362.

Patapy, C., Huger, M., Guinebretière, R., Gey, N., Humbert, M., Hazotte, A. \& Chotard, T. (2013). J. Eur. Ceram. Soc. 33, 259268.

Petit, J., Castelnau, O., Bornert, M., Zhang, F. G., Hofmann, F., Korsunsky, A. M., Faurie, D., Le Bourlot, C., Micha, J. S., Robach, O. \& Ulrich, O. (2015). J. Synchrotron Rad. 22, 980-994.

Plancher, E., Favier, V., Maurice, C., Bosso, E., Rupin, N., Stodolna, J., Loisnard, D., Marijon, J.-B., Petit, J., Micha, J.-S., Robach, O. \& Castelnau, O. (2017). J. Appl. Cryst. 50, 940-948.

Plancher, E., Petit, J., Maurice, C., Favier, V., Saintoyant, L., Loisnard, D., Rupin, N., Marijon, J.-B., Ulrich, O., Bornert, M., Micha, J.-S., Robach, O. \& Castelnau, O. (2016). Exp. Mechanics, 56, 483-492. Poulsen, H. F. (2012). J. Appl. Cryst. 45, 1084-1097.

Rauch, E. F. \& Dupuy, L. (2005). Arch. Met. Mater. 50, 87-99.
Riekel, C. (2000). Rep. Prog. Phys. 63, 233-262.

Robach, O., Kirchlechner, C. et al. (2014). Strain and Dislocation Gradients from Diffraction, edited by R. I. Barabash \& G. E. Ice, pp. 156-204. Imperial College Press/World Scientific Publishing.

Robach, O., Micha, J. S., Ulrich, O., Devincre, B., Hoc, T., Daveau, G., Consonni, V. \& Petit, J. (2017). Rayons X et Matière 5, edited by R. Guinebretière \& P. Goudeau, pp. 83-142. London: ISTE Editions Ltd.

Robach, O., Micha, J.-S., Ulrich, O., Geaymond, O., Sicardy, O., Härtwig, J. \& Rieutord, F. (2013). Acta Cryst. A69, 164-170.

Robinson, I. \& Harder, R. (2009). Nat. Mater. 8, 291-298.

Sibil, A., Douillard, T., Cayron, C., Godin, N., R'mili, M. \& Fantozzi, G. (2011). J. Eur. Ceram. Soc. 31, 1525-1531.

Smirnov, M., Mirgorodsky, A. \& Guinebretière, R. (2003). Phys. Rev. $B, \mathbf{6 8}, 104106$

Spolenak, R., Brown, W. L., Tamura, N., MacDowell, A. A., Celestre, R. S., Padmore, H., Valek, B., Bravman, J. C., Marieb, T., Fujimoto, H., Batterman, B. W. \& Patel, J. R. (2003). Phys. Rev. Lett. 90, 096102.

Tamura, N. (2014). Strain and Dislocation Gradients from Diffraction, edited by R. I. Barabash \& G. E. Ice, pp. 125-155. Imperial College Press/World Scientific Publishing.

Tamura, N., Celestre, R. S., MacDowell, A. A., Padmore, H., Spolenak, R., Valek, B. C., Meier Chang, N., Manceau, A. \& Patel, J. R. (2002). Rev. Sci. Instrum. 73, 1369-1372.

Ulrich, O., Biquard, X., Bleuet, P., Geaymond, O., Gergaud, P., Micha, J. S., Robach, O. \& Rieutord, F. (2011). Rev. Sci. Instrum. 82, 033908.

Ungár, T., Castelnau, O., Ribárik, G., Drakopoulos, M., Béchade, J. L., Chauveau, T., Snigirev, A., Snigireva, I., Schroer, C. \& Bacroix, B. (2007). Acta Mater. 55, 1117-1127.

Villert, S., Maurice, C., Wyon, C. \& Fortunier, R. (2009). J. Microsc. 233, 290-301.

Wan, V. V. C., Cuddihy, M. A., Jiang, J., MacLachlan, D. W. \& Dunne, F. P. E. (2016). Acta Mater. 115, 45-57.

Wilkinson, A. J., Meaden, G. \& Dingley, D. J. (2006). Ultramicroscopy, 106, 307-313.

Zhang, F. G., Bornert, M., Petit, J. \& Castelnau, O. (2017). J. Synchrotron Rad. 24, 802-817.

Zhang, F. G., Castelnau, O., Bornert, M., Petit, J., Marijon, J. B. \& Plancher, E. (2015). J. Appl. Cryst. 48, 1805-1817. 